\title{
The Impact of Domestic Plug-in Hybrid Electric Vehicles on Power Distribution System Loads
}

\author{
Sikai Huang, and David Infield, Senior Member, IEEE
}

\begin{abstract}
The market for Plug-in Hybrid Electric Vehicle (PHEVs) is expected to grow significantly over the next few years and a number of new products are soon to come onto the market, such as the Toyota Prius plug-in version, [1]. The charging demand of wide-scale use of PHEVs may have a significant impact on domestic electricity loads and could risk of overloading the power system if appropriate charging strategies not applied to prevent this. A Monte Carlo Simulation (MCS) model of domestic PHEV use and availability has been developed based on probabilistic characterisations obtained from UKTUS and quantifies charging demand of PHEVs as a function of time of day. The MCS model has been developed in order to simulate the impact on the electricity distribution system. This article also discusses the potential for responsive battery charging load from PHEVs.
\end{abstract}

Index Terms--Demand Side Management, Responsive Load, Plug-in Hybrid Electric Vehicle, Power System Operation, Electricity Distribution System, Vehicle-to-Grid.

\section{NOMENCLATURE}

$N C$ - number of cars in TUS sample population

$\mathrm{NH}$ - number of households in TUS sample population

$P N(I)$ - probability of numbers of cars in a household, where the random variable $I$ is the number of cars: $0 \sim 6$

$P A_{j}$ - probability of an PEV arriving back home at time interval $j$

$P J L_{i}(T)$ - probability distribution for journey time $T$ (in units of 10 minutes) for journeys arriving back home at time interval i

$Q J L_{i}(T)$ - cumulative probability distribution for journey time $T$ (in units of 10 minutes) for journeys arriving back home at time interval $\mathrm{i}$

$Q N(I)$ - cumulative density function for numbers of cars $I$ per household

$T_{\text {drive }}$ - journey duration

$E_{\text {drive }}$ - energy used for a particular journey

$U_{\text {drive }}$ - average driving speed

$k$ - energy consumption coefficient

$T_{\text {charge }}$ - battery charging duration

$P_{\text {charging_level }}-$ power taken from the charger

$\eta$ charging - charging efficiency

This work was supported in part by UK Science and Engineering Research Council grant EP/F022832/1.

S. Huang and D. Infield are with the Department of Electronic Electrical Engineering, University of Strathclyde, Glasgow, G11XW, UK. (e-mail: sikai.huang@eee.strath.ac.uk).

\section{INTRODUCTION}

$\mathrm{T}$ ransport is a significant source of domestic carbon emission, representing 21 per cent of total UK domestic carbon emission, where passenger cars take part of 58.3 per cent, [1]. The UK Government has set a carbon emission reduction target of $80 \%$ by 2050 based on 1990 level. The UK electricity network operator National Grid (NG) has announced that it plans to reduce carbon emission by $45 \%$ by $2020,[3]$. Interest in the electric transport sector, especially hybrid electric vehicles (HEV) and plug-in electric vehicles (PEV) has increased dramatically in recent years. PEVs, including Battery Electric Vehicles (BEV) and Plug-in Hybrid Electric Vehicles (PHEV), offer great opportunity to both reduce the fuel consumption and carbon emissions by using electricity as its main source of power. For the automobile industry, electricity is a cheaper alternative to oil based fuels, which has the benefits of carbon emission reduction and reduced operational costs. The market of BEVs and PHEVs has been quickly growing over the past few years. Battery technologies specifically for HEVs and PEVs have been developed that are more cost effective, with more robust charging/discharging approaches, and higher efficiencies. Major car manufacturers such as General Motors (GM), Honda, BMW MINI, and Toyota, all have HEV and PEV models available, either as prototypes or actual products. Some excellent EVs are already on the market, such as the Toyota Prius, Honda Insight, Tesla Roadster, G-Wiz and others. However, following the early market success of HEVs, PHEVs have the most potential for wide-scale adoption by domestic consumers in the next decade, and this will require additional electricity utility supply, Distribution Network Operators (DNO), direct involvement to optimise the use of PHEVs in order to prevent overloading the system. While PHEVs has the potential social and economic benefits, reliable and safety operation of the electrical network is still the predominant utility concern.

Detail analysis of current UK domestic car use can help to understand and accurately prediction the use of PHEVs and their electrical system impacts; this is important for electricity utilities as well as automotive manufactures. There is one particular concern that much of the additional battery charging load may occur at times when the electricity supply system is already heavily loaded and will impact across the entire power system. It is likely that PHEVs will not be evenly distributed geographically due to their limited range will make them more attractive in urban areas rather than rural areas. In the early stage of deployment, it is fairly possible that some cities will have higher penetrations of PHEVs as a result of car 
manufacture leasing programmes and incentive schemes, or local planning that encourage public to use PHEVs or BEVs instead of conventional vehicles, Internal Combust Engine (ICE) vehicle, for instance, London has a incentive schemes of free congestion and parking fees.

A number of studies of BEVs and PHEVs impact have been published, for example references [4]to [9], these are often based on simplistic assumptions and not estimated the uncertainties of PEVs charging demand as a result of the stochastic nature of vehicle use. EPRI has lunch a multi-year study of impact of PEVs and PHEVs on utility distribution systems, and papers [4] and [5] illustrate the potential impact of PEVs on distribution system, such as substation transformers and system losses. Veltman, [6], emphasises the hidden flexibility of PEVs battery charging loads and thus utilised in the context of demand side management (DSM). Larsen and colleagues at DTU/Riso in Denmark, [7], are exploring how this flexibility could be used to ease the integration of high penetrations of wind generation in power system and their economic benefits for providing vehicle-togrid (V2G) power. Hadley at Oak Ridge National Laboratory, US, [8] and [9], has concentrated on PHEVs. Except PHEVs have much more small battery capacity and limited range than BEVs, they are currently taking a larger market share, and also are more considerable make charging impact on the power distribution system in the short term. As BEVs and PHEVs have on board batteries used as DESD, a growing interest in using them to provide ancillary services, supporting peak demands. There are some examples, [10] to [13], present the use of PEVs as a source of distributed generation to support power system operation. This is beyond the scope of the research reported in this article where the concern is on presenting a methodology for detailed PHEVs battery charging load calculation. It would be possible to apply the model developed here to V2G applications and this has been considered for future research topic.

\section{Domestic Vehicle Use Data And Stochastic Model DEVELOPMENT}

A Monte Carlo method has been selected in modelling domestic car use due to its potential to quantify uncertainties associated with expected outcomes. Sequential Monte Carlo Simulations (MCS) are used to evaluate potential impacts on distribution network based on projections of certain aspects of PHEVs proliferation/use. This stochastic approach is intended to capture temporal and spatial diversity of PHEV integration. MCS models must be underpinned by large volumes of relevant data in order to obtain reliable and credible results. For this study, domestic car use data has been extracted and classified from the UK Time of Use Survey (UKTUS) 2000, [14]. This survey is based on diaries filled by thousands of individual domestic participants describing their daily activities with a 10 minutes time resolution. The specific method data was recorded enables unambiguous identification of privately owned car movements, the purpose of journeys and the location where the car is parked when it is not being driven on the road. This data base has been analysed to derive probability distributions required as inputs for the MCS modelling.

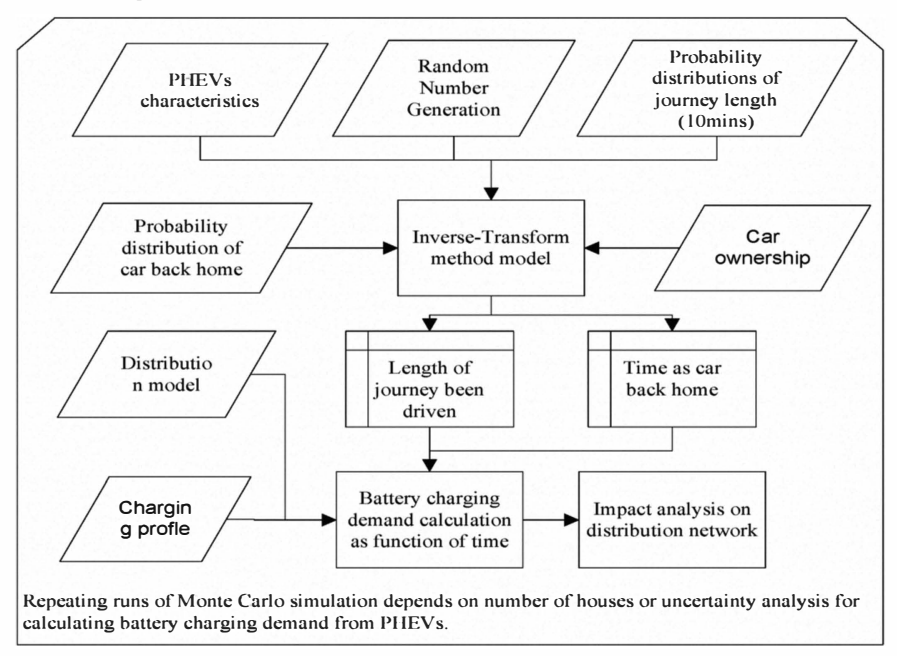

Fig. 1. Monte Carlo simulation model structure.

\section{A. UK Vehicle Statistics}

In National Travel Survey (NTS) 2009, it states that there were 34.2 million licensed vehicles registered in Great Britain. There are approximately 28 million cars licensed, with $89 \%$ of them privately owned, [15]. TUSUK data base provides detail information of domestic car use, and this information have been interrogated to obtain series of probability distributions, covering time of use, distance of journey, location of parking, time of parking, etc. Weekdays and weekends have been distinguished for analysis and modelling purposes due to their contrasting characteristics. The obvious morning and evening peaks can be distinctly identified in the weekday analysis, reflecting a combination of work commute and possibly the "school run". Between these two peaks, majority of the car population are parked either at working place or home. Journeys starts later during weekend, this is expected from involvement of much more extended use of domestic cars.

Most of the time these cars are parked; they are on average being driven for only about $5 \%$ of the time. These parked cars, $96 \%$ of the time, could thus provide a valuable secondary function as responsive load. This figure is in agreement with [16], where it is shown that average personal vehicle in use is only $4 \%$ of the day leaving $96 \%$ of the day when it is not in use. More details of the statistics covering household car ownership and overall domestic car use derived from TUSUK 2000 can be found in Huang and Infield, [17]. This article presented here makes use of 5,137 diaries covering 3,606 households.

\section{B. Monte Carlo Method for Impact Analysis of PHEVS Charging Demand}

In the first analysis, it is assumed that battery charging starts as the time when PHEVs arrive home since this is the most likely and secure approach from domestic car users' point of view. PHEVs charging levels vary from $120 \mathrm{VAC}$ to $240 \mathrm{VAC}$, and charging power from $1.2 \mathrm{~kW}$ to $15 \mathrm{~kW}$. There is also a specific quick charging level using a 3 phase supply, 
Table 1. Toyota Prius Plug-in Hybrid Vehicle and Regular Hybrid Vehcile Configurations

\begin{tabular}{lrr}
\hline & Regular version & Plug-in version \\
\hline Battery Capacity $(\mathrm{kWh})$ & 1.31 & 5 \\
Battery type & Nickle-Metal Hydride & Lithium-ion \\
Nominal voltage (V) & 201.6 & 345.6 \\
Number of battery modules & 28 & 288 \\
Range limits - electric only mode (miles) & 1.2 & 13 \\
Top speed (mph) & 30 & 60 \\
Charging period (hours) & 0 & 1.7 \\
Charging level (kW) & & 3.12 \\
\hline
\end{tabular}

and its charging power is larger than $15 \mathrm{~kW}$. However, this type of charger would be expected to equip public car parking lots rather than domestic houses. Maitra and his colleagues, [18], have discussed the potential electrical demand over time, or charge profile, defined by the battery size and charge type. In this work, it is simply assumed that PHEVs charge at a steady rate until fully charged, when on-board battery charging stops. PHEVs have a significantly different vehicle configuration from existing conventional Internal Combustion Engine (ICE) vehicles. PHEVs consume both petroleum and electricity as their driving fuel. Plug-in hybrid technology uses electricity from the utility grid to charge on board battery and reduce petroleum consumption beyond that of Hybrid Electric Vehicles (HEV). PHEVs also contribute to carbon emission reduction of transport sector. There are two types of PHEVs.

With wide scale roll out of PHEVs in the next decades, charging demand from PHEVs acts as additional load on top of the domestic demand and will bring vital impacts on the power distribution network. It is important to understand the ramifications of adding load from PHEVs onto the grid. Depending on when and where the vehicles are plugged in, they could cause local or regional constraints on the grid. They could require the addition of new electric capacity and increase the utilization of existing capacity.

Constant charge rate is a fairly reasonable assumption and it can be easily modified to the taper charge algorithms as used when batteries approach being fully charged. Table 1 shows the Toyota Prius 2009 version and 2011 plug-in version. Toyota Prius has been a successful hybrid vehicle (HV) model for the last decades. The details of new plug-in version provide sufficient information for calculating battery charging demand. All PHEVs are assumed identical and are based on Toyota Prius plug-in version, [19], as shown in Table 1. At the present stage of PHEVs' market development, this is a sensible assumption. Variation of future PHEVs characteristics will fairly quickly average out with small overall impact on the aggregate charging loads. However, the capacity of the battery and the charging level will definitely have a vital impact on overall charging loads. Local penetrations of PHEVs will determine in the main the magnitude of the additional load on the distribution network. In the UK, distribution networks are at $11 \mathrm{kV}$ down to 400 Volt and their assets are the 3 Phase transformers, with domestic load supplied at 230 Volt. The approach in this model is making use of probability distributions of the car being driven back home and length of the total round trip journey. These probability distributions are used as the input of MCS model. It is obvious that the probability of a car being driven and especially the probability that a journey set off from home at a given time interval will depend on the time of day as well as on the probability distribution of journey length. These are then clearly conditional probability distributions: dependent on the time of day. In practice though the MCS modelling constructed for this research proceeds forward in time and thus at any particular time step, computations use only the simple probability density function associated to the time step in question.

The discrete probability density function (pdf), $P N(I)$, for different number of cars $I$, allows the MCS model to allocate different number of vehicles in each households. Fig. 2 below shows the pdf for individual household obtained from the TUSUK data base and for the population of 4,972 households. As a result of modelling, a number of households have no cars; the total number of cars in the sample population was 5,158 , giving on average 1.037 cars per households.

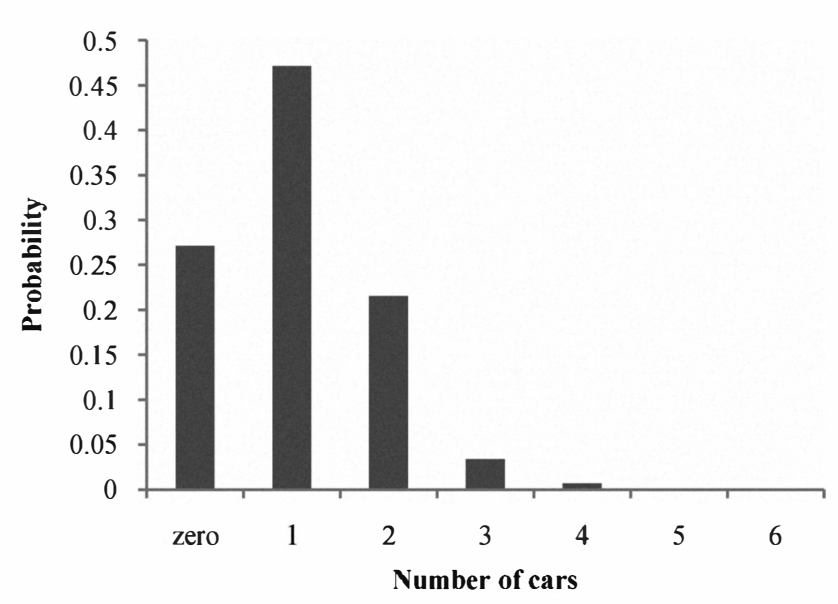

Fig. 1. Household car ownership distribution as derived from UKTUS 2000

This information is critical for later stage research. The exact location of houses will be required in order to perform detailed power flow calculations in the distribution network case study. In the TUSUK data, domestic vehicle use have been defined as conventional vehicle, ICE vehicle; however, in the MCS modelling development different penetrations are assumed to have been replaced with PHEVs. In the modelling of length of journey, car been driven as back home, have been classified to suit for PHEVs characteristics. For example, if 
the total length of journey as when the car returns back home is less than its limited range, on-board battery is discharged as PHEV been driven under electric-only mode; otherwise, if journey is longer than the range limit, and it is assumed that battery returns with half its capacity. This is a reasonable assumption given that one the battery is charged from the ICE it will cycle between fully charged and fully discarded ${ }^{1}$ and greatly simplifies the analysis.

For each ten minute time interval throughout 24 hours, the probability of a given car arriving back home, $P A_{\mathrm{i}}$, is calculated for all weekday data, directly from:

$$
P A_{i}=\text { number of cars arriving back home at time step } i / N C
$$

$P A_{i}$ is plotted against time of day in Fig. 3.

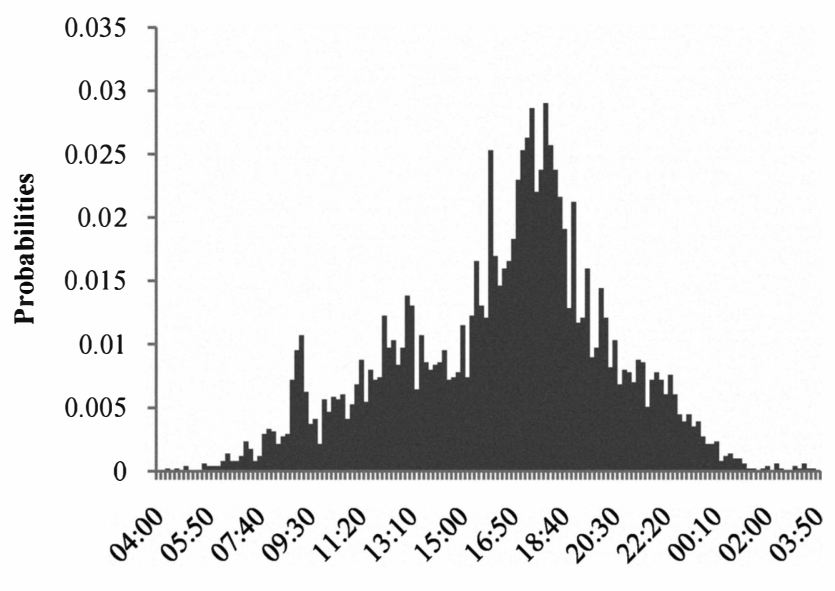

Time (24hrs)

Fig. 2. Probabilities of arriving home throughout a typical weekday

For each time interval $i$, a single probability distribution of journey length, $P J L_{i}(T)$, used to calculate the likely distance travelled by each PHEV when it arrives back home, as all cars are treated the same. $T$, represents the time duration, is a discrete random variable and equals to the number of ten minute periods. Length of journey is only accurate to the nearest 10 minutes due to the diaries TUSUK recorded. If a car has only been driven between 5 minutes, it will be recorded as a 10 minutes journey. It is necessary to have the consistent with the TUSUK data. In [20], some example distributions are presented for journeys ending as car back home at different times of day. It is important to understand that these probabilities are for total round trip driving times instead of period of time that car been driven away from home.

As previous described, TUSUK provides information relative to the duration of each journey; however, they are all based on driving time rather than distance travelled. MCS is performed to identify when domestic cars have been driven back home and as a proportion of PHEVs take-up, their onboard battery state of charge (SOC) status. SOC is used in calculation of charging time required to bring battery to either fully charged or partial charged. For simplicity, a constant

\footnotetext{
${ }^{1}$ Fully discharged is a nominal definition and will not signify the battery has zero charge remaining, since this would damage the battery.
}

rate of charge is used and PHEV users start to charge their cars immediately on arriving home. In this study, it is only considered that PHEVs charge at home. The procedure of the MCS model to calculate round trip driving times is described in detail in [20].

\section{Calculation of PHEVs charging load on Distribution System}

Once the analysis of total driving times been established, it is straightforward to calculate the corresponding degree of battery discharge for each returning PHEV by assuming reasonable average urban driving speed and energy consumption based on the PHEV model described in the previous context. The average urban driving speed used in this study is $30 \mathrm{mph}$ and is based on the results reported in, [15]. It is noticeable that PHEVs' limited range varies as a result of driving speed. In the modelling, driving behaviour has changed when PHEVs take in place of conventional vehicles. Calculation of the total energy consumed also requires knowledge of the vehicle performance in terms of energy consumed per unit time at this average speed. The energy consumption coefficient for a typical urban driving cycle for the Toyota Prius plug-in version is $0.23 \mathrm{kWh} / \mathrm{mile}$, [19]. This vehicle has a practical range limit of 13 miles; therefore, recharging period of time is calculated as method described previous conditions, reflecting an assumption that all longer journeys would make use of a conventional vehicle. The total energy used $E_{\text {drive }}$ for a particular journey of duration $T_{\text {drive }}$ is calculated as follows:

$$
E_{\text {drive }}=T_{\text {drive }} \bullet U_{\text {drive }} \bullet k
$$

where $U_{\text {drive }}$ is the average driving speed (30 $\mathrm{mph}$ in these calculations), and $k$ is the energy consumption coefficient $(0.23 \mathrm{kWh} / \mathrm{mile})$.

For example, if a PHEV drives for period of time of 10 minutes (1/6 hours), it will consume $1.15 \mathrm{kWh}$ according to these assumptions. For the type of electric vehicle chosen in the simulation, the battery capacity is $5 \mathrm{kWh}$ with only $60 \%$ of that capacity available for driving, so as not to fully discharge the battery at any stage. Assumed that all PHEVs recharge batteries as immediately on return to the house, as well as at a fixed charging rate until battery is fully charged, it is straightforward to calculate the charging duration $T_{\text {charge }}$ (in hours) from:

$$
T_{\text {charge }}=\frac{E_{\text {drive }}}{P_{\text {charging_level }} \bullet \eta_{\text {charging }}}
$$

where $P_{\text {charging_level }}$ is the rating of the charger taken as $2.99 \mathrm{~kW}$ based on the specification of the charger: 13 Amps at 230 Volts, [5]. The charging efficiency $\eta_{\text {charging }}$ is assumed to be $0.90,[5]$.

The urban distribution network, detailed in [22], of a complete $11 \mathrm{kV}$ feeder and all associated LV (400/230 V) networks including six distribution transformers, providing 
power to 1,262 connected customers, has been used as a guide to a distribution system supplying predominantly domestic housing. Each distribution transformer in this system supplies 214 houses. Initially all 1,262 households are considered together so as to examine the additional charging load on the substation transformer. With such a large sample of houses the Monte Carlo simulation only needs to be run once to give a reasonable estimate of the mean additional loads. In this work, it is necessary to understand the aggregate charging loads associated with domestic load.

According to the car ownership statistics, these 1,262, houses will have 1,735 cars, and 20 percent of these are initially assumed to be PHEVs. Fig. 4 gives the calculated additional $\mathrm{EV}$ charging load as a function of time of day for an EV take-up rate of $20 \%$, and is shown together with a typical daily load profile for UK domestic loads taken from UKGDS, [23], but suitably scaled to the number of houses supplied. The mean load for a single household according to UKGDS profile is 580 Watts; this is very close to the figure of 536 Watts available from DUKES for 2009, [24].

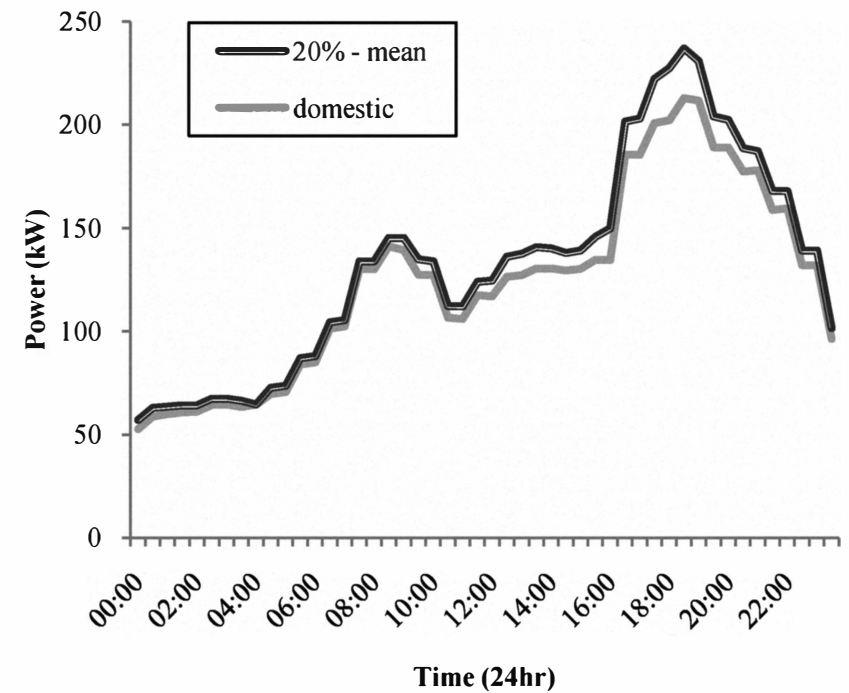

Fig. 4. Additional load due to PHEV charging for a population of 1262 houses and an assumed EV take up of $20 \%$.

It should be noted that the load information has a time resolution of 30 minutes, reflecting the UK market arrangements. For comparison purposes only the 10 minute Monte Carlo results have been averaged up in 30 minute blocks. It is apparent that for this UK example the charging load occurs very much at the time of the existing peak load. This is unsurprising but the magnitude of the new peak is significant, even for the modest PHEV ownership assumed for this case.

Comparing to large sample simulation, attention is now focused on a single group of 214 houses supplied by a single distribution transformer and with PHEV take up, as before, of $20 \%$. It has been expected to be some variation between individual simulation runs with this smaller sample size. To identify the mean behaviour but more importantly to gain an understanding of the uncertainties associated with the additional load, the simulation must be re-run numerous times with different random number seeds, which has been implemented in MATLAB. Reasonable convergence was achieved with 100 simulation runs. Fig. 5 shows a representative sample of the results for 3 of the 100 runs; the solid line shows the mean charging load as a function of time of day. The ten minute time resolution has been maintained here.

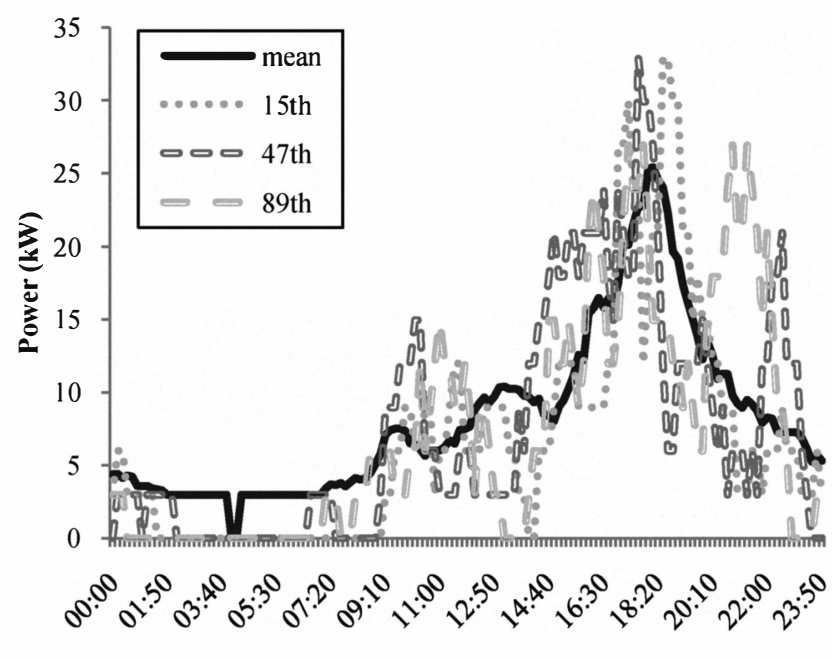

Time (24hr)

Fig. 5. Example results of 100 runs of Monte Carlo simulation for Electric vehicles charging demand for 214 houses with $20 \%$ PHEVs take-up.

Fig. 6 brings together the expected electricity load profile for a typical weekday with the expected electric vehicle charging load and its uncertainty. This shows that the maximum additional load at the relatively low EV penetration of $20 \%$ is very significant (more than $30 \%$ ) in comparison with the conventional domestic peak demand and that both of these occur at almost exactly the same time. In order to examine the impact of differing EV penetrations, the analysis of the 214 houses has been re-run but with penetrations of $20 \%, 30 \%$ and $50 \%$ as shown in Fig. 7 . Only mean charging loads are show, so that following the above discussion, peak values will be considerably greater.

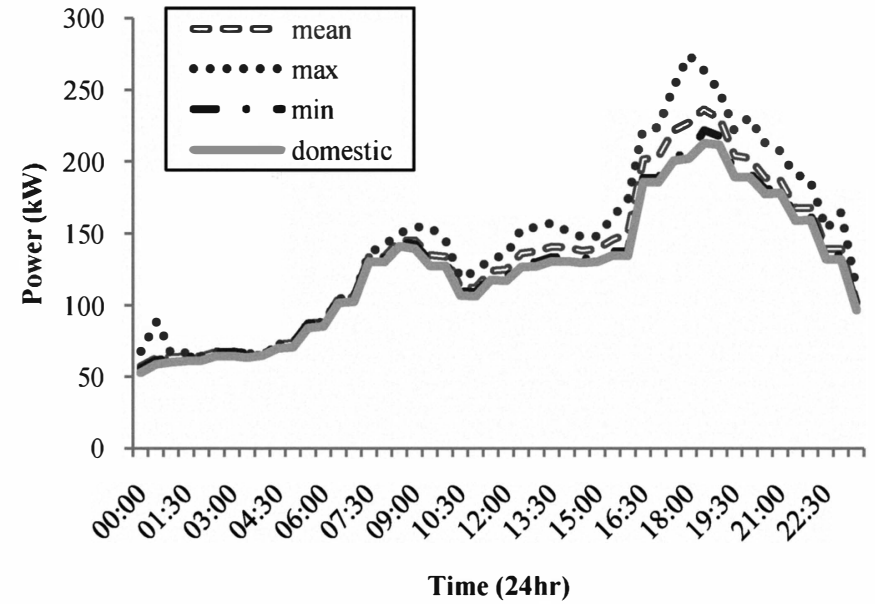

Fig. 6. Domestic PHEVs charging demand with expected range compared with aggregated domestic load. 


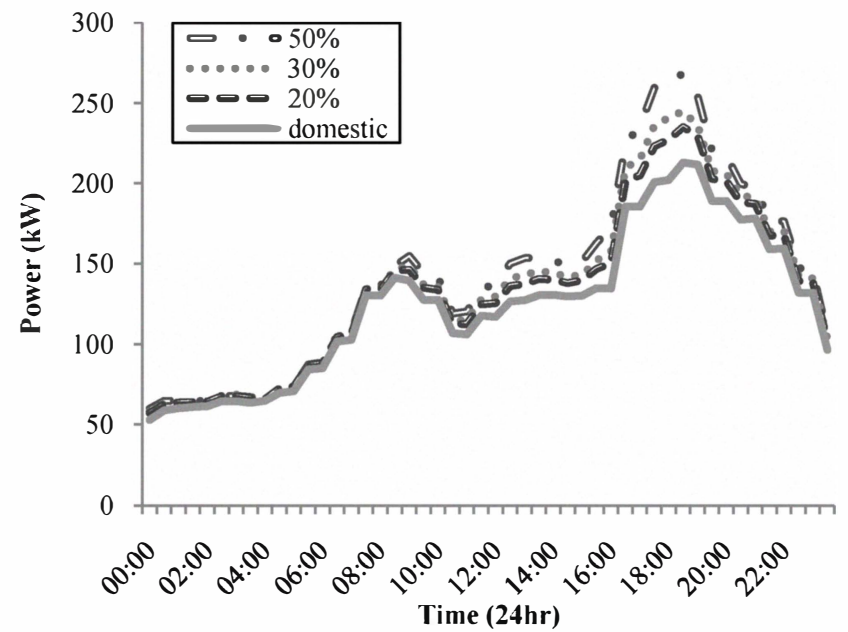

Fig. 7. Domestic PHEVs mean charging demand as responsive load comparing with aggregated domestic load; with $20 \%, 30 \%, 50 \%$ EV take up levels.

\section{CONCLUSION}

Wide-scale domestic use of PHEVs will certainly have impact on the power distribution system design and operation. A Monte Carlo method for modelling PHEV charging load has been presented in detail. Based on TUS UK Survey 2000, typical UK domestic car use information has been obtained, where details of car travelling pattern for both weekdays and weekends cover time of use, duration of use and distance travelled as well as car ownership in each household. Analysis of the UK domestic car use data shows that private owned cars are occupied by driving on the road only for $5.2 \%$ of the time, theoretically $94.8 \%$ of the remaining time providing opportunity for load shifting or secondary function, such as V2G. Application of the method to the typical UK urban LV network shows that distribution transformers and undoubtedly associated distribution lines will need substantial upgrading if any significant deployment of PHEVs. The methodology presented in this paper can be regarded as a useful tool for Distribution Network Operators (DNOs) and also be used as an input to detailed power flow analysis. As future research involves use of the United Kingdom Generic Distribution System (UKGDS), which provides a shared resource for the description and simulation of distribution network models that are representative those found in the UK. Together with daily and annual domestic load profiles these results can be used to investigate the potential impacts on the distribution network of different penetrations of PHEVs. It should be noted that in the work presented here no attempt at "smart" charging has been made; PHEVs are charged immediately on return to the home. There is clearly enormous scope for retiming of the charging to better suit overall power system operation, thus acting as responsive load providing demand side services. The MCS model presented will be used to explore these issues in future work.

\section{ACKNOWLEDGMENT}

This work was supported by the Engineering and Physical Sciences Research Council, UK, project numbers EP/F022832/1 and E.ON UK. The authors would like to thank Murray Thomson and Ian Richardson at Loughborough University for their support, and also the researchers within the Renewable Energy Technologies Group at Strathclyde.

\section{REFERENCES}

[1] EDF Energy, Toyota and EDF Energy launch the UK's largest plug-in hybrid vehicle leasing programme, http://www.edfenergy.com.

[2] The Secretary of State for Transport, Low Carbon Transport: A Greener Future, A Carbon Reduction Strategy for Transport, July 2009.

[3] UK National Grid to cut carbon emissions 45 pct by 2020 , Committee on Climate Change. [Online]. Available:

http://www.theccc.org.uk/news/climate-change-news/256-uk-nat-gridto-cut-carbon-emiss ions -45-pct-by-2020.

[4] A. Maitra, et al., "Integrating plug-in- electric vehicles with the distribution system," in 20th International Conference and Exhibition on Electricity Distribution (CIRED 2009), 8-11 June 2009, Stevenage, UK, 2009, p. 1029 (5 pp.).

[5] J. Taylor, et al., "Evaluation of the impact of plug-in electric vehicle loading on distribution system operations," in Power \& Energy Society General Meeting, 2009. PES '09. IEEE, 2009, pp. 1-6

[6] E. Veldman, et al., "Unlocking the hidden potential of electricity distribution grids," in 20th International Conference and Exhibition on Electricity Distribution, CIRED 2009, June 8, 2009 - June 11, 2009, Prague, Czech republic, 2009.

[7] E. Larsen, et al., "Electric Vehicles for Improved Operation of Power Systems with High Wind Power Penetration," in Energy 2030 Conference, 2008. ENERGY 2008. IEEE, 2008, pp. 1-6.

[8] S. W. Hadley, "Evaluating the impact of Plug-in Hybrid Electric Vehicles on regional electricity supplies," in Bulk Power System Dynamics and Control - VII. Revitalizing Operational Reliability, 2007 iREP Symposium, 2007, pp. 1-12.

[9] S. Hadley, "Impact of Plug-in Hybrid Vehicles on the Electric Grid," Oak Ridge National Laboratory ORNL/TM-2006/554, October, 2006.

[10] J. A. Pecas Lopes, et al., "Using vehicle-to-grid to maximize the integration of intermittent renewable energy resources in islanded electric grids," in Clean Electrical Power, 2009 International Conference on, 2009, pp. 290-295.

[11] W. Kempton and J. Tomic, "Vehicle-to-grid power fundamentals: calculating capacity and net revenue," Journal of Power Sources, vol. 144, pp. 268-79, 2005.

[12] W. Kempton and J. Tomic, "Vehicle-to-grid power implementation: From stabilizing the grid to supporting large-scale renewable energy," Journal of Power Sources, vol. 144, pp. 280-94, 2005.

[13] J. Tomic and W. Kempton, "Using fleets of electric-drive vehicles for grid support," Journal of Power Sources, vol. 168, pp. 459-68, 2007.

[14] The United Kingdom 2000 Time Use Survey Technical Report 2003, [Online]. Available:

http://www.statistics.gov.uk/downloads/theme_social/UKTUS_TechRep ort.pdf.

[15] Department for Transport (DfT), Transport Statistics: Great Britain 2009 Edition 35th Edition ed.: TSO (The Stationery Office), 2009.

[16] W. Kempton, et al, "Vehicle-to-Grid Power: Battery, Hybrid, and Fuel Cell Vehicles as Resources for Distributed Electric Power in California", UCD-ITS-RR-01-03, June, 2001.

[17] S. Huang and D. G. Infield, "The potential of domestic electric vehicles to contribute to power system operation through vehicle to grid technology", Proceedings of the 44th International Universities Power Engineering Conference, 2009.

[18] A. Maitra, et al., "Integrating plug-in- electric vehicles with the distribution system," in 20th International Conference and Exhibition on Electricity Distribution (CIRED 2009), 8-11 June 2009, Stevenage, UK, 2009, p. 1029 (5 pp.)

[19] Toyota, 2010 Prius Plug-in Hybrid Makes North American Debut at Los Angeles Auto Show, pressroom.toyota.com, December, 2009. 
[20] D. G. Infield and S. Huang, "Impact of Electric Vehicle Charging on the Distribution System", submitted to IEEE Transactions on Power Systems.

[21] R. Y. Rubinstein and D. P. Kroese, Simulation and the Monte Carlo Method. (2nd ed.). New Jersey: Wiley, 2008, p. 52.

[22] M. Thomson and D. G. Infield, "Impact of widespread photovoltaics generation on distribution systems," IET Renewable Power Generation, vol. 1, pp. 33-40, 2007.

[23] United Kingdom Generic Distribution System (UKGDS), http://monaco.eee.strath.ac.uk/ukgds/

[24] BERR, DUKES 2009, available online at: http://www.decc.gov.uk/en/content/cms/statistics/publications/dukes/duk es.aspx 